\title{
Space Maintenance by Hemisection of Primary Mandibular Second Molar: An Alternate Treatment Approach
}

\author{
Rutika Lalwani ${ }^{1}$, Adesh Kakade ${ }^{2}$, Sheetal Badnaware ${ }^{3}$, Sayali Mali ${ }^{4}$ \\ Doccumenttype: Case Report \\ ${ }^{1}$ Post graduate student, Department of Pediatric and Preventive Dentistry, Nair Hospital Dental College, \\ Mumbai \\ ${ }^{2}$ Professor and Head of Department, Department of Pediatric and Preventive Dentistry, Nair Hospital Dental \\ College, Mumbai \\ ${ }^{3}$ Post graduate student, Department of Pediatric and Preventive Dentistry, Nair Hospital Dental College, \\ Mumbai \\ ${ }^{4}$ Post graduate student, Department of Pediatric and Preventive Dentistry, Nair Hospital Dental College, \\ Mumbai
}

\begin{abstract}
As the premature loss of primary mandibular second molar results in space loss, a space maintainer is always indicated to prevent this space loss. However, there are certain disadvantages to the conventional fixed nonfunctional space maintainers in respect to the longevity of the appliance, undesirable effects on the abutment and the opposing tooth and patient compliance. The purpose of this case report was to discuss an alternative treatment option for space maintenance by hemisection of the fractured primary mandibular second molar followed by a cast crown restoration. The crown restoration was casted to reproduce the natural anatomy of the primary mandibular second molar and contact with the erupting permanent molar was established to aid in its proper eruption into occlusion.
\end{abstract}

Keywords: Space maintainers, Hemisection, Primary mandibular second molar.

\section{Introduction}

Guidance of eruption and development of the primary, mixed, and permanent dentitions is an integral component of comprehensive oral health care for all pediatric dental patients. Such guidance should contribute to the development of a permanent dentition that is in a stable, functional, and esthetically acceptable occlusion and normal subsequent dentofacial development. Early diagnosis and successful treatment of developing malocclusions can have both short-term and long- term benefits while achieving the goals of occlusal harmony and function and dentofacial esthetics. ${ }^{1,2}$

The developmental phases of dentition as well as its eruption sequence, symmetry, and chronology should be known by the practitioner, in addition to managing leeway space for proper positioning of permanent dentition

The premature loss of primary teeth due to caries, trauma, or other causes may lead to undesirable tooth movements of primary and/or permanent teeth including loss of arch length. Arch length deficiency can produce or increase the severity of malocclusions. ${ }^{3}$

Treatment of developing malocclusion in a growing child is important as it not only benefits the child psycho-socially but also normalizes the perioral musculature which is conducive to the normal growth of dental arches, jaws and the face. ${ }^{4}$ The malocclusion when left untreated usually gets complicated and more difficult to treat by conventional means.

Emphasis must always be to use the deciduous teeth as "natural space maintainers".However, in cases where it is not feasible to preserve the tooth, a space maintainer must always be considered after the extraction of the deciduous tooth and a careful review of the patient should be maintained.

Premature loss of primary mandibular second molar almost always results in space $\operatorname{loss}^{5}$ and thus poses a challenge to maintain this space for further development of proper occlusion.

\section{Case report}

An 7year 6 month old male child reported to the OPD with the chief complaint of pain in the lower left back region. On intra oral examination there was a partially dislodged restoration along with the fracture of the crown of primary mandibular left second molar. His medical history was noncontributory. On obtaining the complete past dental history, it was revealed that pulpectomy procedure was performed with the same tooth 3 months back, and the patient did not report for the post endodontic stainless steel crown restoration.Radiograph with the associated tooth revealed properly filled root canals. The crack line could not be seen on the radiograph, probably due to the superimposition of the intact tooth structure. 
On completely removing the fractured restoration,fracture line was visible clinically (figure 1). A decision was made to remove the fractured fragment. The patient was prepared for the treatment under Local Anaesthesia (2\% Lignocaine infiltration). The coronal fragment was completely removed. On observing the fractured fragment, it was noticed that the fracture line extended to the cervical third of the distal root. Since the mesial half of the tooth showed no radiographic signs of endodontic failure, the hemisection of the distal half of the tooth was planned. The tooth was sectioned buccolingually using a fissure bur and the tooth was split open into two segments. The remaining part of the distal root fragment was then extracted using extraction forcep. Post-operative bleeding was controlled. The exposed portion of the crown was then sealed with a glass ionomer cement restoration. The extraction wound was allowed to heal.

On immediate post-operative follow up the next day, the patient was completely asymptomatic. After 1 week, the wound area showed satisfactory healing process with no signs of infection and inflammation. At the end of 15 days, a study model impression with alginate was made for the upper and lower arch. After thorough examination of the cast and radiographic evaluation, a cast crown restoration was planned. The purpose of the crown was to serve as a functional space maintainer to prevent the space loss which can occur by the distal root extraction. The hemisectioned primary second molar was restored with Composite resin restoration. The crown preparation was done for full coverage cast metal crown (figure 2). A post preparation alginate impression was made.Wax mockup was done on the cast for full coverage cast metal restoration involving the space created after the hemisection. Care was taken to establish proper contact with permanent $1^{\text {st }}$ molar to maintain the space.Casting was done for the wax pattern and final finishing and polishing was done.

The crown was seated into the mouth and minor adjustments into the occluion were done after checking it with an articulating paper. Themargins of the crown were checked for proper marginal fit and the crown was cemented using type I glass ionomer cement (figure 3). The excess cement was removed using an explorer and the occlusion was checked. A post-operative radiograph was taken to evaluate the proper seating of the crown (figure 4 ).

\section{Discussion}

Childs dentition is a dynamic entity,which keeps on changing as the transition from primary to mixed and mixed to permanent dentition occurs. Premature loss of primary second molar during the mixed dentition period almost always results in space loss. Thus it becomes mandatory to maintain this space for harmonious eruption of the permanent teeth and maintenance of occlusion. Space maintenance often fails due to poor appliance design, poor case selection and lack of patient compliance and cooperation. ${ }^{6}$ It has been stated that space closure will occur maximally during first six months of the tooth loss and also during the active eruption of the permanent molar. ${ }^{7}$

Literature shows that there is significant mesial migration of the mandibular permanent molar during its eruption. This can lead to loss of space created by premature loss of primary second molar.For premature loss of primary mandibular second molar during the active eruption of the mandibular first permanent molar, a reverse band and loop space maintainer is a conventional treatment being followed. ${ }^{8}$ However, during this phase, the eruptive forces are concentrated on the reverse band and loop appliance, which can lead to the failure and also may hinder the harmonious eruption of the permanent tooth.Other disadvantages reported with this space maintainer are breakage at the solder joint, soft tissue impingement, rotation of the abutment tooth. Also there is inadequate crown length available for banding and also the anatomy of deciduous first primary molar may make an adequate fit band placement difficult. It needs to be replaced by a conventional band and loop space maintainer once the permanent molar is in complete occlusion. This adds to the cost and compliance of the patient. $^{9,10}$

It has been documented in the literature that the failure rate for band and loop space maintainer varies between $29 \%$ to $37 \% .{ }^{10}$ However,no long term studies have been reported with reverse band and loop in the literature.Thus, considering the above factors, in this case we planned a cast crown restoration on the hemisected primary mandibular second molar. A proper broad and flat contact was reproduced on the casted crown. A broad and flat contact created by the cast crown provided a proper eruption guidance to the actively erupting permanent molar. Thus preventing its mesial migration resulting in maintenance of the arch length and establishment of the harmonious occlusion.Also a crown in place will help in mastication unlike a nonfunctional space maintainer, and also helps in preventing the supraeruption of the opposing tooth. Preserving the tooth in the arch has an added advantage of the preservation of the alveolar height and also aids in the timely eruption of the succedaneous tooth.Every attempt should be made to preserve the natural tooth in the oral cavity with maintaining the natural dimensions and establishing the contacts to prevent changes in the arch length. 


\section{References}

[1]. Kanellis MJ. Orthodontic treatment in the primary dentition. In: Bishara SE, ed. Textbook of Orthodontics. Philadelphia, Pa: WB Saunders Co; 2001:248-56.

[2]. Woodside DG. The significance of late developmental crowding to earlytreatment planning for incisor crowding. Am J Orthod Dentofacial Orthop. 2000;117(5):559-61

[3]. Tunison W, Flores-Mir C, ElBadrawy H, Nassar U, El-Bialy T. Dental arch space changes following premature loss of primary first molars: a systematic review.Pediatr Dent. 2008;30(4):297-302

[4]. Wright GZ, Kennedy DB. Space control in the primary and mixed dentitions. DentClin North Am. 1978;22(4):579-601.

[5]. Rönnerman A. The effect of early loss of primary molars on tooth eruption and space conditions. A longitudinal study. Acta Odontol Scand. 1977;35(5):229-39.

[6]. Terlaje RD, Donly KJ. Treatment planning for space maintenance in the primary and mixed dentition. ASDC J Dent Child. 2001;68(2):109-14, 80

[7]. Ngan P, Fields H. Orthodontic diagnosis and treatment planning in the primary dentition. ASDC J Dent Child. 1995;62(1):25-33

[8]. Arora B, Setia V, Chauhan I, Mahajan M, Nangia T. Reverse Band And Loop-When And Where To Give???. Indian J Dent Sci. 2014;6(3):62-65.

[9]. Gellin ME, Spedding RH. Space management required after unsuccessful rootcanal therapy of a mandibular second primary molar: case report. Pediatr Dent.1990;12(4):253-6.

[10]. Sasa IS, Hasan AA, Qudeimat MA. Longevity of band and loop space maintainers using glass ionomer cement: a prospective study. Eur Arch Paediatr Dent. 2009;10(1):6-

\section{FIGURES:}

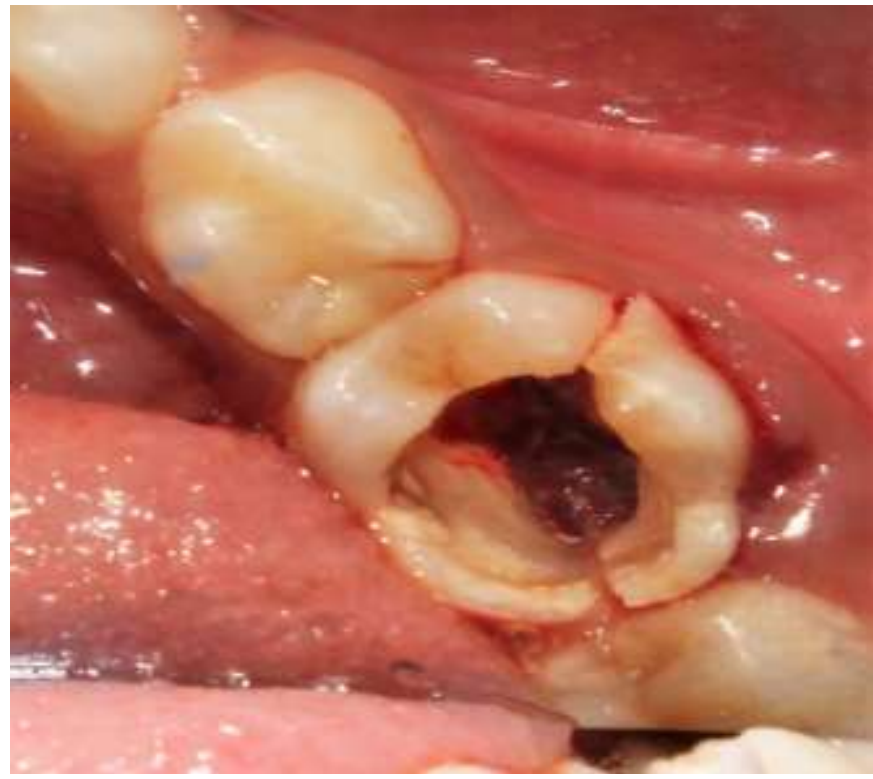

FIGURE 1: Pre-operative view showing fracture line after removal of the fractured restoration

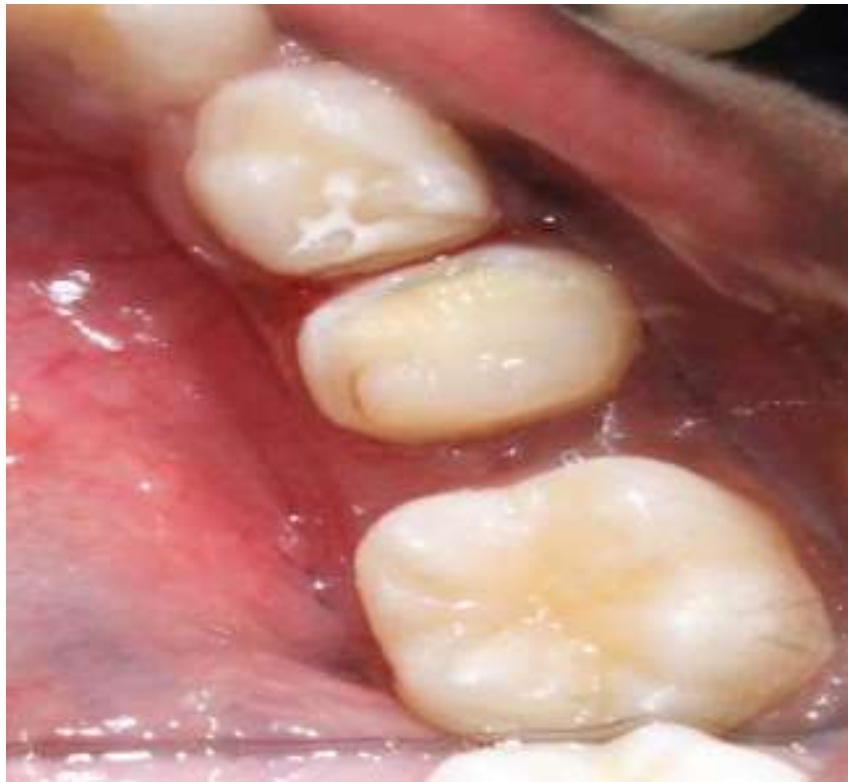

FIGURE 2: Composite restoration of the hemisected primary mandibular second molar 


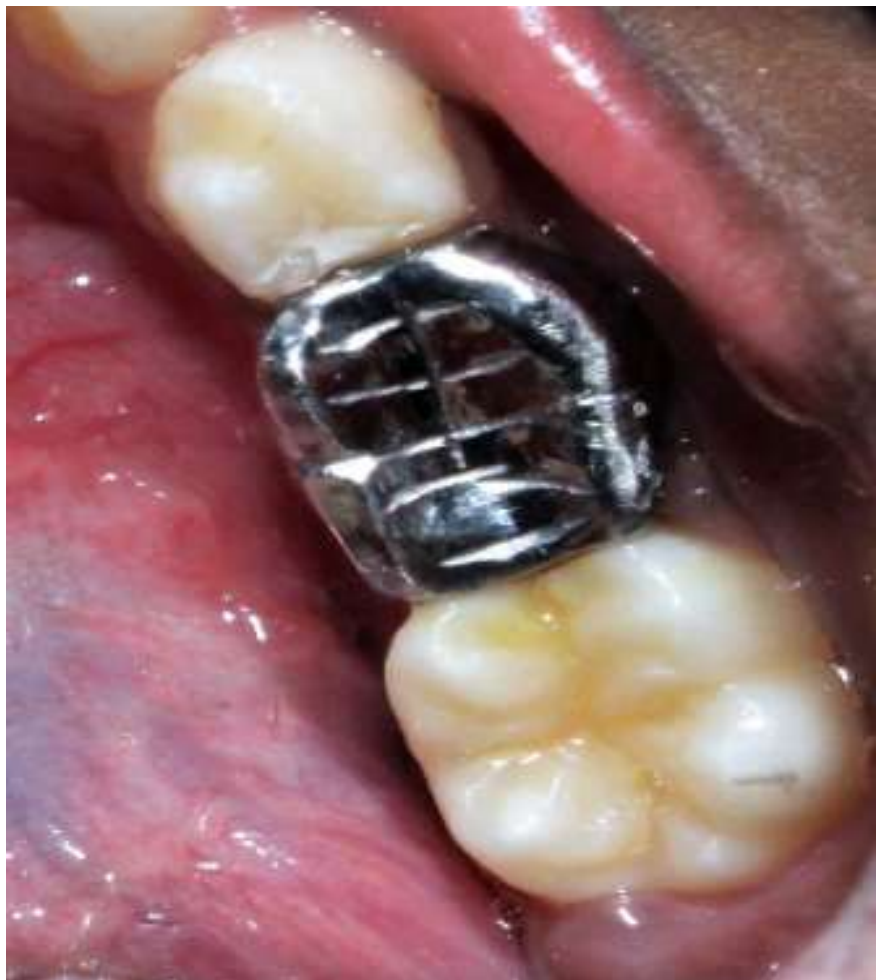

FIGURE 3: Post-operative view after cementation of the cast crown restoration

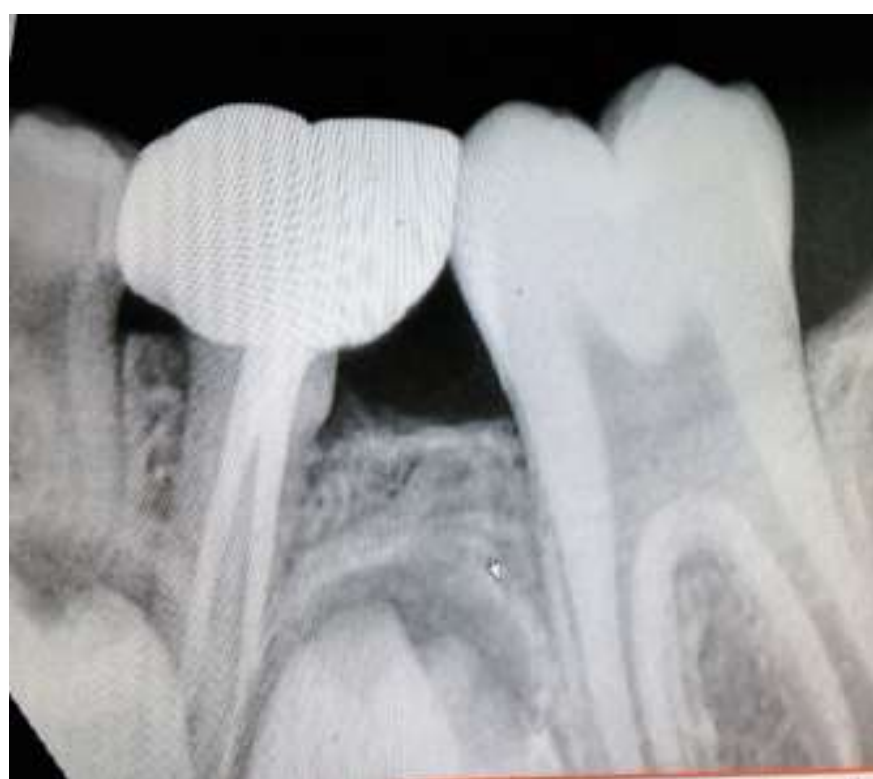

FIGURE 4: Post-operative radiograph 